\title{
Introduction to Special Issue on RC 13 Sessions at the 2018 International Sociological Association World Congress
}

\author{
Shintaro Kono ${ }^{1} \cdot$ Karl Spracklen $^{2}$ \\ Published online: 21 February 2019 \\ (C) Springer Nature Switzerland AG 2019
}

What characterizes the sociology of leisure? This question came to his mind when the first author was attending approximately 80 presentations delivered at Research Committee 13 (RC13) Sociology of Leisure sessions at the 19th International Sociological Association (ISA) World Congress of Sociology in Toronto, Canada, in 2018. We believe that part of the answer lies in its "diversity." Presenters defined, analyzed, and discussed leisure from diverse cultural and national perspectives. They also presented on varying forms of leisure practices around the world, including sports, tourism, outdoor recreation, spirituality, events, festivals, technology, art and craft, music, and games. Presenters were not only leisure scholars and sociologists by training, but also tourism and sport scholars, economists, social psychologists, health researchers, anthropologists, and so on. Their scholarly techniques were also diverse ranging from highly theoretical work to empirical (qualitative, quantitative, and mixed) work. The sociology of leisure is diverse in its cultural, disciplinary, and scholarly underpinning. In this special issue based on such various conference presentations, the following papers also represent this rich diversity.

Before describing each paper, we would like to describe the background of the current special issue. The International Journal of the Sociology of Leisure (IJSL) is the official journal affiliated with RC13 sociology of leisure at the ISA. It published its first volume in 2018. RC13 has been the space where leisure scholars, sociologists and others exchange their works since 1961. In 1956, Joffre Dumazedier proposed to form a

This manuscript is an introduction to the special issue on RC 13 Sessions at the 2018 International Sociological Association World Congress.

Shintaro Kono

Shintaro.kono@siu.edu

1 Department of Public Health and Recreation Professions, College of Education and Human Services, Southern Illinois University Carbondale, Pulliam Hall 312, 475 Clocktower Drive, Carbondale, IL 62901, USA

2 School of Social Sciences, Leeds Becket University, Leeds, UK 
special committee under the ISA focused on leisure. Since then, RC13 has had renowned leisure scholars as its executive members, such as Stan R. Parker, Kenneth Roberts, Robert Stebbins, Jiri Zuzanek, Francis Lobo, and Ishwar Modi. For the 19th ISA congress, RC13 organized 14 sessions on a variety of topics and received approximately 150 abstract submissions. The presentations that survived the peerreview process exhibited both excellent diversity and rigor.

As the first special issue of the IJSL, we are proud to present the following nine articles that represent diverse cultural, disciplinary, and scholarly perspectives. The first paper, entitled "Unequal Leisure Opportunities Across Genders - Overwhelmed Women” by Klára Tarkó and Zsuzsanna Benkö, reveals how women in Hungary are deprived of leisure time across socio-demographic groups (e.g., age, residential location, labor market status, and education status). They do so by using three waves of nationally representative time budget survey data between 1986 and 2010. Gender gaps in the work life domain is well known in the public (e.g., 20\% less salary for the comparable positions among female workers compared to their male counterparts; United States Census Bureau 2017). Tarkó and Benkő's paper offers the fundamental information based on which we can further educate the public about gender issues in a leisure life domain, as well as develop research, practice, and policies to address the problems. Indeed, Tarkó and Benkö's findings seem consistent with the data from, for example, Canada (Moyser and Burlock 2018). What is unique to the current special issue paper is that it (a) examines the intersection between gender and other sociodemographic factors, (b) inspects gender gaps in time spent on different types of leisure activities (e.g., socialization, media use, exercise and sports), and (c) adds insight from an Eastern European country.

The second paper, entitled "Playing in the Shadow of Event Urbanism Newcomer Youth, Neighborhood Change, and TO2015" by Amanda De Lisio, Gregory Yerashotis, and Caroline Fusco, examines the intersection between urban space experience among newcomer youth and sport mega events in Toronto, Canada. Specifically, the authors conducted a critical qualitative analysis of documents as well as observations and semi-structured interviews with the youth within the context of the $2015 \mathrm{Pan} /$ Parapan American Games (TO2015). Their analysis revealed that event organizers associated the event venue space with lack of productivity and presence of criminality, and that they reimagined it as more productive, safer, and healthier place through the sport events. Simultaneously, newcomer youth were often forced to either participate in the events as volunteers and other productive members or leave the space altogether. Anti-social behavior and communities were increasingly censored and excluded from the urban area. The events were also used as an opportunity to idealize active, elite sport participants as the model image of citizen. While reporting these critical empirical findings, De Lisio et al.'s work also engages with immigrants as an important population in the globalizing world as well as mobilizes insight from multiple disciplines, such as critical geography and sport sociology.

The third paper, entitled "The Digital Nomad Lifestyle: (Remote) Work/Leisure Balance, Privilege, and Constructed Community" by Beverly Yuen Thompson, analyses the phenomenon called digital nomad lifestyle or "the ability for individuals to work remotely from their laptop and use their freedom from an office to travel the world." This fascinating topic clearly challenges the conventional boundary between work and leisure. Moreover, the subject also speaks to other relevant fields, such as 
tourism and media studies. Thompson analyses qualitative data collected through participant observations and in-depth interviews. In her analysis, Thompson utilizes both mainstream sociological concepts (e.g., privilege, commodification) as well as leisure-specific concepts (i.e., Stebbins's serious leisure). The paper illustrates a complex life of digital nomads: while many are taking advantage of their privilege (e.g., gender, passport power, English-speaking ability), they often felt separated from local culture and people in the host society and sought "authentic" relationships beyond commodified communities through conferences and social media. Their motivation to develop a community appears particularly strong among non-traditional, disenfranchised nomads (e.g., young females).

The fourth paper, entitled "Sapucaí Street: Entertainment Hub and Commercial Gentrification in Belo Horizonte" by Clarissa dos Santos Veloso and Luciana Teixeira de Andrade, is an ethnographic study of a street called Sapucaí in the city of Belo Horizonte, Brazil. What is interesting about this particular urban space is that since 2012, it has experienced a rapid development of leisure establishments, such as restaurants and bars, by micro-business owners. Although once it was a mere outskirt boarder of the city, today the street attracts a number of relatively young customers and creates a vibrant nightlife scene. Dos Santos Veloso and de Andrade pointed out, based on their observation and interview data, that this development was a form of commercial gentrification, which differs from the traditional, residential gentrification. That being said, the authors also contended that these business owners did not enjoy absolute power over their business area and clientele. Indeed, as the area became more popular, different types of clients joined, who may enjoy hedonic music rather than critiques and discussion of a movie and cause noise and nuisance. The paper illustrates the transient, dynamic nature of power at play in a particular urban street in Brazil.

The fifth paper is entitled "An Analysis of Transformations in the Mass Media Constructions of Black Women's Hair through Leisure Reading: A Case Study of Drum Hair Magazine" by Aretha Oluwakemi Asakitikpi and Miliswa Tamara Choene. Although it is well documented and discussed that human bodies, especially female bodies (e.g., "bikini body"), are under close scrutiny in contemporary media, the authors shed light onto another body part that has been severely understudied: hair. They content-analyzed words and images used in a popular hair magazine mainly for Black women called Drum Hair Magazine in South Africa. Asakitikpi and Choene argued, "Through the leisure reading activity of Black hair care magazines, Black women are encouraged to use various haircare products to transform their natural hair texture into one that is long and straight in order to better 'manage' and have a more 'acceptable' image/identity." The researchers also noted a recent trend to re-emphasize the importance of "natural hair"; however, they were quick to point out that the very word "natural hair" was juxtaposed with images of non-natural hair, such as straightened hair, hair extensions, and even wig. This paper illustrates yet another way that the leisure industry censors our bodies, exerts power over people especially those in social minority groups, and influences our identity.

The sixth paper, entitled "Developments in Information Technology and the Sexual Depression of Japanese Youth Since 2000" by Maki Hirayama, analyze potential reasons for inactive sexual life or "sexlessness" among Japanese youths. Sex in general has been a largely neglected topic within broader leisure studies (Berdychevsky 2018). Yet, this study further pushes sex-related leisure research agendas by exploring this 
peculiar phenomenon in the Eastern Asian country. Based on national surveys, Hirayama first established the fact that Japanese youths - especially junior high school, high school, and university students - participated in dating, sexual kiss, and sex less frequently especially since 2005 . The researcher, then, discussed five factors related to information technology that could have contributed to this sexless trend: communication via email and social media, dating websites and phone applications, sexual service industry, online pornography, and fantasy and beautification among otaku or pop culture geeks in Japan. Hirayama used a comprehensive review of relevant studies and national survey data to support each of her arguments. Furthermore, her arguments reflected social, cultural, legal, economical, historical, and political background of Japan, and thus exemplified how a universal topic like sex can differ across nations. For instance, some Western readers may wonder why social media, dating websites, and phone applications can contribute to the decrease in sexual activities, not increase. This is partly because, Hirayama maintained, these technologies had been long used for "compensated dating" in Japan where young females had sex with older men for money. Thus, the technologies did not foster genuine interest in and interaction for romance and sexual relationships.

The seventh paper, entitled "Forecasting the Social Return on Investment Associated with Children's Participation in Circus-Arts Training on Their Mental Health and WellBeing" by Richard McGrath and Kristen Stevens, also represents the diversity of topical areas and methodological choices within the sociology of leisure. The researchers examined the effects of circus-art program participation on children's mental health and well-being, by adopting a mixed-methods design based on pre- and postsurveys and focus group. Although the quantitative results did not reach statistical significance, the qualitative findings revealed perceived improvements in self-esteem, interpersonal skills, enjoyment, and stress coping. Moreover, McGrath and Stevens took a step further to identify the amount of money invested in the circus-art program and to quantify the psycho-social benefits of this program, so that they could clarify the cost-effectiveness of the program. To this end, they used an analytic approach called the Social Return on Investment (SROI). Their results showed that for every one dollar spent on the program, children participants gained seven-dollar worth psycho-social benefits. This is an exciting line of evidence that has direct implications for leisure service practice and leisure-related policies. McGrath and Stevens's research shows us the fact that something is difficult to quantify should not stop us from engaging with practically and theoretically important questions. Sociologists of leisure must continue practically and politically relevant research, as well as theoretical projects.

The eighth paper, entitled "Cities Regulated by Cultural Events: Tracking Music Festivals in Lisbon and São Paulo" by Paulo Nunes, examines what roles music festivals play in an urban life in the context of the Virada Cultural in São Paulo, Brazil and the Mexefest in Lisbon, Portugal. To address this issue, Nunes employed a combination of direct observation, literature review, and document analysis, as well as Actor Network Theory. On the one hand, the study found that these music events were used to promote the cities' brand image and increase their tourist attraction. Another important finding was that these festivals provided the space for social tolerance and inclusion, while also causing substantial crowding and gentrification. For example, some of music events in Portugal represented disenfranchised social groups such as immigrants, refugees, and LGBTQ people, within their programs. 
However, the city government used the Mexefest and other large-scale events as an excuse for "revitalizing" and developing the urban space where the festivals took place, which resulted in gentrification and exclusion of socially marginalized groups. Moreover, Nunes argued that event organizers and the city of São Paulo utilized the Virada Cultural for the purpose of social control. Specifically, they attempted to "decentralize" social and cultural capital by hosting events at different outskirt areas of the city, where controversial issues such as graffiti and fights between competing groups of rappers were integrated. Thus, this paper shows us how complicated social consequences of musical festivals can be in metropolitan cities. They may liberate and marginalize social groups at the same time. We are reminded that leisure is neither panacea to social problems nor source of mere social oppression; leisure is complicated.

The ninth paper is entitled "Household Expenditure on Leisure: A Comparative Study of Italian Households with Children from Y- and Z-Generation" by Simona Diliberto, Michele Tumminello, and Fabio Massimo Lo Verde. In this study, the researchers engaged with leisure consumption - the important topic that interests not only sociologists of leisure, but also economists and other scholars - within a specific population: Italian families with teenagers. Specifically, they conducted a secondary analysis of nationally representative survey data from 2001, 2007, and 2012, by using cluster and logistic analyses. Doing so allowed them to separate the effects of different time eras and generations of children (i.e., the 2001 and 2007 data for Generation Y, while the 2012 data for Generation Z). Their results suggested that both different time eras (and thus different social situations) and generations of children had effects on families' leisure consumption patterns. For example, comparing the 2001 and 2012 data, they found that families with Generation-Y children spent more on outdoor recreation (e.g., canoeing, windsurfing) than their counterparts with Generation- $Z$ children. On the other hand, leisure consumption patterns among families with Generation- $Z$ children were characterized by the expenditure on a variety of activities including arts and culture (e.g., dance, painting, music). Diliberto et al. speculated that this may have been because some skills and interests related to outdoor activities eroded among newer generations, while technologically savvy children introduced different types of leisure activities to their families. However, these generational differences disappeared when the 2007 and 2012 data were compared, supposedly because the time lapse between these two periods was smaller and thus social environment would be similar. In the latter case, it seemed that other social-demographic factors such as income and education level shaped families' leisure patterns. Their logistic regression also identified newspaper and analog photography as significant predictors of families' having Generation-Y children. This finding may mean that Generation-Z children who were born with and grew up with new digital technology stayed away from the traditional forms of media. Collectively, these results suggest the existence of both within- and across-generation differences in leisure consumption. Thus, Diliberto et al.'s work seems to agree with other papers in this special issue that the sociology of leisure is a complex phenomenon, which rejects the "either/or" perspective.

In conclusion, this special issue on RC 13 Sessions at the 19th ISA World Congress represents the remarkable diversity within the sociology of leisure. Culturally, the papers in this issue represent each of the five continents. Topically, their foci ranged from the classic issue of gender imbalance in leisure time and leisure consumption 
patterns, to emerging, controversial topics such as sexlessness and digital nomad lifestyle. Methodologically, they encompassed qualitative, quantitative, and mixedmethods. However, the special issue papers also seem to converge on some key themes: that leisure is the space where both social justice and injustice manifest; that leisure changes dynamically as society changes; that studying leisure requires a variety of theoretical and methodological skills; and that leisure matters. We hope that these papers will inspire more diverse and exciting ideas within readers' mind.

Publisher's Note Springer Nature remains neutral with regard to jurisdictional claims in published maps and institutional affiliations.

\section{References}

Berdychevsky, L. (2018). "Risky" leisure research on sex and violence: Innovation, impact, and impediments. Leisure Sciences, 40(1/2), 9-18.

Moyser, M., \& Burlock, A. (2018). Women in Canada: A gender-based statistical report. Retrieved from https://www150.statcan.gc.ca/n1/en/pub/89-503-x/2015001/article/54931-eng.pdf?st=4XVAZlj2. Accessed 18 Feb 2019.

United States Census Bureau. (2017). Income and poverty in the United States: 2016. Retrieved from https://www.census.gov/content/dam/Census/library/publications/2017/demo/P60-259.pdf. Accessed 18 Feb 2019. 